\title{
24.
}

\section{Eigenschaften und Beziehungen der Ausdrücke, welche bei der Auflösung der allgemeinen cubischen Gleichungen erscheinen.}

(Von Herrn Stud. G. Eisenst ein zu Berlin.)

Als ich im ersten Hefte des 27 ten Bandes dieses Journals die allgemeine Auflösung der Gleichungen von den ersten 4 Graden mittheilte, versprach ich, bei einer andern Gelegenheit auf die Eigenschaften der in die Auflösungsformeln eingehenden Ausdrücke zurückzukommen. Vielleicht wird es einigen Lesern nicht unangenehm sein, wenn ich hier dieses Versprechen wenigstens in Beziehung auf die cubischen Gleichungen erfülle.

Die Aufgabe, eine allgemeine cubische Gleichung aufzulösen, ist identisch mit derjenigen, die homogene Function

$$
a x^{3}+3 b x^{2} y+3 c x y^{2}+d y^{3}=\Phi
$$

in ihre 3 linearen Factoren zu zerfällen. Man kann diese Zerfällung auf doppelte Weise ausführen, je nachdem man nämlich in der Gleichung

$$
\boldsymbol{\Phi}=0
$$

entweder $x$ oder $y$ als die Unbekannte ansieht. Im ersten Falle findet man, dafs $\boldsymbol{a}^{2} \Phi$ in das Product dreier Factoren zerfällt, von denen jeder die Form

$$
\text { 1. } a x+\left(b+v^{3}\left(\frac{1}{2}\left(a_{1}+a \sqrt{D}\right)\right)+3^{3}\left(\frac{1}{2}\left(a_{1}-a \sqrt{D}\right)\right)\right) y
$$

hat, und wo die Werthe der Cubikwurzeln so zu nehmen sind, dafs ihr Product

$$
=A=b^{2}-a c
$$

wird.

Im zweiten Falle erhält man die Zerlegung von $d^{2} . \Phi$ in das Product dreier linearer Factoren von der Form

$$
\text { 2. } \quad d y+\left(c+\sqrt[3]{ } /\left(\frac{1}{2}\left(d_{1}+d_{V} D\right)\right)+{ }^{3}\left(\frac{1}{2}\left(d_{1}-d_{V} D\right)\right) x,\right.
$$

wo das Product der Cubikwurzeln

$$
=C=c^{2}-b d
$$

sein mufs. 
Die Werthe von $a_{1}, d_{1}, D$ sind

$$
\begin{gathered}
a_{1}=a^{2} d-3 a b c+2 b^{3} \\
d_{1}=d^{2} a-3 d c b+2 c^{3} \\
D=a^{2} d^{2}-3 b^{2} c^{2}+4 a c^{3}+4 d b^{3}-6 a b c d .
\end{gathered}
$$

$\mathrm{Zu}$ den beiden Ausdrücken $a_{1}$ und $d_{1}$ gesellen sich noch die beiden andern

$$
\begin{aligned}
& b_{1}=a b d-2 a c^{2}+b^{2} c, \\
& c_{1}=d c a-2 d b^{2}+c^{2} b,
\end{aligned}
$$

und zu $\boldsymbol{A}$ und $\boldsymbol{C}$ der folgende:

$$
\boldsymbol{B}=\boldsymbol{b} \boldsymbol{c}-\boldsymbol{a d} .
$$

Diese Ausdrücke von $a_{1}, b_{1}, c_{1}, d_{1}, A, B, C$ und $\boldsymbol{D}$ bilden zusammen mit den Coëfficienten $\boldsymbol{a}, \boldsymbol{b}, \boldsymbol{c}, \boldsymbol{d}$ ein in sich abgerundetes Ganze.

Wenn man aus den Ausdrücken von $-a_{1},-b_{1}, c_{1}$ und $d_{1}$ vier neue auf dieselbe Art entstehen läfst, wie jene aus $a, b, c, d$ entstanden sind, so erhält man resp.

$$
-a D^{2},-b D^{2},-c D^{2},-d D^{2} .
$$

Die Ausdrücke $a_{1}, b_{1}, c_{1}, d_{1}$ werden aus den partiellen Differentialquotienten von $\boldsymbol{D}$ nach $d, c, b, a$ gefunden, wenn man diese letzteren resp. mit

multiplicirt.$$
\frac{1}{2},-\frac{1}{6},-\frac{1}{6} \text { und } \frac{1}{2}
$$

Die drei Formen

$$
\begin{gathered}
a x^{3}+3 b x^{2} y+3 c x y^{2}+d y^{3}=\Phi \\
-a_{1} x^{3}-3 b_{1} x^{2} y+3 c_{1} x y^{2}+d_{1} y^{3}=\Phi_{1}, \\
A x^{2}+B x y+C y^{2}=\boldsymbol{F}
\end{gathered}
$$

hangen auf das innigste mit einander zusammen. Wenn man auf alle drei eine lineare Substitution

deren Nenner

$$
\begin{aligned}
& x=\lambda x^{\prime}+\mu y^{\prime} \\
& y=\nu x^{\prime}+\rho y^{\prime}
\end{aligned}
$$

$$
\lambda \rho-\mu \nu=1
$$

ist, anwendet, so wird man das merkwürdige Verhalten wahrnehmen, dars zwischen den Coëfficienten der drei neuen Formen $\Phi^{\prime}, \Phi_{1}^{\prime}$ und $F^{\prime \prime}$ in $x^{\prime}, y^{\prime}$ genau dieselben Relationen Statt finden, wie zwischen denen von $\Phi, \Phi_{1}, F$ : ein Umstand, der besonders eine wichtige Grundlage für zahlentheoretische Untersuchungen bildet. 
Der Ausdruck $\boldsymbol{D}$, den man Determinante von $\Phi$ nennen kann, ist zugleich die Determinante von $\boldsymbol{F}$, weil

$$
\boldsymbol{D}=\boldsymbol{B}^{2}-4 \boldsymbol{A C}
$$

ist. Die Determinante von $\Phi_{1}$ ist dann $D^{3}$. Diese Determinante bleibt bei jeder linearen Substitution, deren Nenner $=1$ ist, unverändert.

Bemerkenswerth sind, aufser mehreren andern, die folgenden identischen Gleichungen:

$$
\begin{aligned}
& 4 A^{3}=a_{1}^{2}-D a^{2}, \quad 4 C^{3}=d_{1}^{2}-D d^{2}, \\
& \boldsymbol{A}^{2}=\boldsymbol{A} b^{2}-B \boldsymbol{B} b+C a^{2} \\
& \boldsymbol{A C}=\boldsymbol{A} \boldsymbol{c}^{2}-\boldsymbol{B} \boldsymbol{b} \boldsymbol{c}+\boldsymbol{C b ^ { 2 }} \text {, } \\
& \boldsymbol{C}^{2}=\boldsymbol{A} d^{2}-B c d+C c^{2} \\
& \boldsymbol{A} \boldsymbol{c}-\boldsymbol{B} \boldsymbol{b}+\boldsymbol{C a}=\boldsymbol{A} d-B \boldsymbol{c}+\boldsymbol{C b}=0, \\
& a_{1}=2 A b-B a \text {, } \\
& b_{1}=A c-C a=+2 A c-B b=+B b-2 C a \text {, } \\
& c_{1}=-A d+C b=-2 A d+B c=-B c+2 C b \text {, } \\
& d_{1}=-B d+2 C c \text {. }
\end{aligned}
$$

Interessant ist noch die Aufgabe, a posteriori die Identität der beiden Zerlegungen (1.) und (2.) nachzuweisen; was ich dem Leser überlasse.

Berlin, 3. März 1844. 